\title{
Urgences
}

\section{Le retour de Don Quichotte ou qui per(d) dure}

\section{Christiane Asselin}

Numéro 26, décembre 1989

Des textes qui chantent

URI : https://id.erudit.org/iderudit/025558ar

DOI : https://doi.org/10.7202/025558ar

Aller au sommaire du numéro

Éditeur(s)

Urgences

ISSN

0226-9554 (imprimé)

1927-3924 (numérique)

Découvrir la revue

Citer cet article

Asselin, C. (1989). Le retour de Don Quichotte ou qui per(d) dure. Urgences, (26), 82-92. https://doi.org/10.7202/025558ar d'utilisation que vous pouvez consulter en ligne.

https://apropos.erudit.org/fr/usagers/politique-dutilisation/ 


\section{Le retour de Don Quichotte ou qui per(d) dure Christiane Asselin}

Le retour de Don Quichotte ${ }^{1}$, sujet de la présente analyse, a été enregistré en direct sur la scène du Spectrum de Montréal, avec la collaboration de la Société Radio-Canada. Il fait partie d'un album double * et comprend des chansons... euh... des monologues... et même quelques applaudissements », nous précise Rivard, sur la pochette du disque. Le retour de Don Quichotte est d'ailleurs ainsi présenté par Rivard, dans ce même album :

Le retour de Don Quichotte s'est fait très... calmement. Le directeur l'a fait v'nir à son bureau, y'a dit: « Vous êtes libre maint'nant, vous pouvez vous en aller. o Fait qu' lui qui était pas plus fou qu'un autr', $y$ ' en a profité pour sacrer son camp. Pis par un beau matin du mois d' mai, y'é r'tourné dans I' quartier qui l'avait vu grandir.

Le retour de Don Qulchotte

1 Je t'écris ces quelques lignes Sur du papier quadrillé

Ça t' rappell'ra l'école et les années folles

Moi c'est le seul papier que j'ai

25 Ça fait déjà deux s'maines

On m'a laissé l' droit d' sortir

Comm' chus pas tell'ment fort su' l' téléphone

J'ai préféré t'écrire

3

$J$ ' te racont' pas tout c' qu'on m'a fait

10 Ni tout c' qu'on m'a fait dire

Ni tout c' qu'on m' a dit pour me fair' croire

Qu'on voulait me guérir

$4 \quad J$ 't'écris pas pour me plaindre

J'avais just' le goût d' parler

1 Le retour de Don Quichotte: paroles et musique: Michel Rivard. Interprète: Michel Rivard. Éditions Bonne Déliwrance, 1985. Tiré du disque mon nom est Michel Rivard et voici mon album double (1985). Étiquette: Kébec-Disc. Distributeur: Diskade Québec. Productions : Alain Simard inc. 
15 C'est encor' troublant pour moi

D'être revenu dans l' quartier

5

Cinq ans sans r'cevoir de nouvelles

Faut dir' qu' j'en ai pas donné

$Y$ ' a des mélodram's qu'on est aussi bien

20 De ne jamais publier

Le soleil me fait tout drôle

Les rues sont bell's ça sent l' printemps

On pourrait peut-êtr' se voir un peu

Peut-êtr' que t'as pas l' temps

7 Ra 25 Tu peux dir' à tout l' monde

Que Don Quichotte est revenu

Avec son ch'fal de porcelaine

Et une armure qui ne tient plus

$8 \mathrm{Rb}$ Les romances impossibles

30 Qui traîn'nt le soir au coin des rues

Comm' les moulins et les géants

$\mathrm{Ne}$ lui font pas plus peur qu'avant

9 Quand on pass' le temps que j'ai passé

A vivr' en attendant

35 Entre les quatre murs et le lit trop dur

Offert par le gouvernement

10 On écrit aux larmes sur les draps blancs

D'incroyables comédies

Pour tous les acteurs drôl's qui appren'nt leur rôle

40 Dans les couliss's de l'ennui

11 Pour tous les fous qui ont peur d' l'amour

Comm' des sirèn's dans la nuit

Pour tout's les fées des étoil's tout's les rob's de bal

De tout's les rein's de tous les carnavals

1245 Pour tous les imbécil's qui chass'nt la baleine

Sur d'immenses bateaux

Mais qui ne voient jamais le poisson d'avril

Qui leur pend dans le dos

$13 \mathrm{Ra}$ Tu peux dir' à tout " monde

50 Que Don Quichotte est revenu

Avec son ch'fal de porcelaine

Et une armure qui ne tient plus 
84

$14 \mathrm{Rb}$ Les romances impossibles

Qui traîn'nt le soir au coin des rues

55 Comm' les moulins et les géants

$\mathrm{Ne}$ lui font pas plus peur qu'avant

15 Comm' tu vois on peut pas vraiment dire

Que quelque chose a changé

Mais je reviens debout c'est déjà beaucoup

60 Et c'est déjà le mois de mai

16

$J$ ' voudrais just' trouver la place

Que tout l' mond' finit par se trouver

Et si t'as l' goût d' me voir

$J$ ' te racont'rai de bell's histoires

65

De chevalier

À l'image du mythique chevalier à la Triste Figure, long, maigre et marginal, le texte de Michel Rivard: seize strophes qui totalisent soixante-cinq vers irréguliers (entre six et huit pieds). Une chanson de cinq minutes cinquante-cinq secondes, durée qui dépasse largement les deux minutes cinquanteneuf habituellement réservés à ce genre d'expression. Un refrain qui ne se répète qu'à une (1) reprise (strophes 13-14) et qui n'est chanté, pour la première fois, qu'à la septième strophe. De même, quatre mélodies différentes et... difficiles. Somme toute, marginale parce qu'elle ne fait pas exactement partie du discours littéraire, cette chanson effectue un second écart à la norme par sa structure générale qui ne correspond pas, comme nous l'avons vu, à celle des autres chansons. Ni tout à fait "poésie", ni tout à fait chanson, presque monologue, parole solitaire d'un être solitaire. D'ailleurs, bien que composé en gamme de do majeur, Le retour de Don Quichotte nous apparaît dramatique. La couleur en est sombre, les déroulements textuel et mélodique lents, un peu comme si l'on assistait à une " reprise" de conscience.

Le retour de Don Quichotte nous laisse sentir cette douleur inexprimable qui perdure depuis Cervantes, celle des mots. * $Y^{\prime}$ a des mélodram's qu'on est aussi bien / De ne jamais publier » (v. 19-20). Et ce sont ces mots, précisément, qui feront l'objet de la présente analyse. Intéressants pour ce qu'ils disent, pour ce qu'ils suggèrent et ce qu'ils taisent aussi. Observons d'abord les références à autrui, dans ce monde clos de l'imaginaire où un homme semble attendre, patiemment (à l'instar de Breton). La 
chanson s'ouvre sur *Je* écrivant à * Tu*: «Je t'écris ces quelques lignes ( $v .1)$. Ce « Tu *, qui revient à douze reprises dans le texte, ce pourrait être Sancho Pança aussi bien que l'auditeur, l'un comme l'autre étant représentatifs du réel, du "bon sens" (si commun). Le «Je», bien sûr, fait référence à Don Quichotte, annoncé dès le titre, ce Don Quichotte qui oppose au réalisme du * Tu * trois autres aspects de l'âme humaine: le rêve, l'idéal, l'amour. On peut compter quatorze références à autrui, sous forme de * eux * ou de * on *, c'est-àdire les fous (v. 41), les fées (v. 43), les imbéciles (v. 45), les guérisseurs (v. 10-12). Les trente occurrences du « Je nous le montrent toujours seul, dissocié d'autrui, perçu ici comme un échec, témoins les vers 9-12, 25-32 et 41-48. En cela Don Quichotte rappelle bien ce mot de Dostoïevski: *Moi je suis tout seul tandis qu'eux ils sont tous.

Le retour de Don Quichotte laisse donc se profiler une impossible communication. La chanson débute par ces mots: «Je t'écris", posant une fois de plus le scripteur en face de lui-même, isolé. Il sera fait mention de la communication sous plusieurs formes à vingt reprises. Cependant, l'écriture se rapporte toujours au * Je»: «Je t'écris * (v. 1); « Jai préféré t'écrire* (v. 8); «J' te racont' pas * (v. 9); «J' t'écris pas pour me plaindre * (v. 13); * On écrit aux larmes * (v. 37); alors que la parole se rapporte à l'autre: * Tu peux dire * (v. 25); * tout c' qu'on m'a dit " (v. 11). Les autres termes ayant trait à la communication sont les suivants: téléphone, nouvelles, mélodrames, publication ( (publier $*$ ), rôle (des acteurs). La parole, événement du présent, n'appartient pas à ce «Je *, venu directement du passé ${ }^{2}$. Un autre phénomène de récurrence baigne cette chanson : celui du temps ${ }^{3}$, qui est nommé à plus de vingt reprises. Ce temps, passé bien sûr (Don Quichotte), mais réactualisé par le biais du retour. Ce temps d'une histoire à poursuivre, et qui hésite entre l'hier et l'àvenir, s'inscrivant toutefois spontanément dans le premier: "les années folles " (v. 3); « Ça fait déjà deux s'maines * (v. 5); «Cinq ans sans r'cevoir de nouvelles * (v. 17), etc.

2 Notons au passage que les rimes croisées, qui touchent systématiquement les deuxième et quatrième vers de chaque strophe, mettent en relief des sons en “é " (parler, publier, quadrillé) et en a ír " (sortir, dire, ecrire, guérir). Ces deux sons font souvent référence aux termes parler et écrire, précisément.

3 Le temps est, lui aussi, marqué par des rimes, cette fois en a an " (printemps, temps, géants, avant, attendant). 
86

Comment lire et lier ces diverses données dans la chanson de Rivard? On peut déjà supposer que Le retour de Don Quichotte crée un lien entre vide et plénitude, parole et musique, silence et cri ou, mieux encore, qu'il « est * ce silence écrit. « Les oiseaux ne parlent pas. Les uns chantent, les autres dévorent... et il se trouve que les dévorés sont toujours ceux qui chantent. ${ }^{4}$ Ceux qui chantent ou ceux qui rêvent? Ce sont, de toute évidence, les mêmes et ils sont marginaux, au même titre que la chanson à l'égard de laquelle on démontre trop souvent un mépris distingué et esthète (ce même mépris qui coiffe la folie). Le retour de Don Quichotte s'inscrit sans relâche dans cette marginalité: chanson au cœur de la littérature, par le sens et l'essence, il traite (sans la nommer, mais en la présentant) de la folie.

Double difficulté donc que celle d'écrire sur l'écriture et sur la folie, par le biais d'une chanson, d'une *parole». Double difficulté par laquelle tout se joue et tout peut se nier aussi : «Ni tout c' qu'on m'a fait dire / Ni tout c' qu'on m'a dit * (v. 10-11) peut s'entendre comme "nier tout *, surtout la parole. Par conséquent, écrire comme ultime recours. Écrire pour tout et pour tous: " Pour tous les acteurs * (v. 39); « Pour tous les fous * (v. 41); «Pour tout's les fées des étoil's tout's les rob's de bal/ De tout's les rein's de tous les carnavals / Pour tous les imbécil's * (v. 43-45). Écrire non plus pour annoncer, mais pour dénoncer, comme le proposent si bien ces vers: * Pour tous les imbécil's qui chass'nt la baleine / [...] / Mais qui ne voient jamais le poisson d'avril / Qui leur pend dans le dos $*$ (v. 45, 47-48). Écrire, pour imposer l'action au cœur même de cette écriture où tout n'est que suspension, attente et désir du rêve. Parler? Jamais autrement que par le biais de l'imaginaire: «J' te racont'rai de bell's histoires / De chevalier " (v. 64-65). Parce qu'elle est fiction, la missive que l'on écrit devient véritablement la « lettre « que l'on chante.

Décidé à laisser vivre son imaginaire, le « Je du Retour est porteur de sens dans sa folie. Il représente, à l'instar du Don Quichotte de Cervantes, l'instrument d'une raison supérieure, toujours en mouvement malgré cinq années de réclusion. Cinq années? Peut-être davantage, car la réclusion n'a pas besoin d'être institutionnalisée, elle est avant tout sociale. Instrument de la pensée, de la raison, "Je " revient donc * debout c'est déjà 
beaucoup" (v. 59). En cela, il se modèle sur la verticalité de la chanson, construite en seize strophes. Verticalisation qui facilite l'image d'un certain triomphe et d'une harmonie certaine. Témoin une autre récurrence: celle du chiffre cinq, symbole de l'homme debout, précisément, symbole de l'univers, du désir d'ordre et de perfection, représentation de la totalité du monde sensible ${ }^{5}$. Ce chiffre apparaît déjà à trois reprises dans la durée de la chanson: cinq minutes cinquante-cinq secondes. Par la suite on le retrouve à cinq reprises dans le texte, directement ou indirectement: «Ça fait déjà deux s'maines " (v. 5), où deux semaines équivalent à quatorze (14) jours, dont la réduction est encore cinq $(1+4)$. Cette indication, comme nous pouvons le constater, se trouve elle-même au vers 5. Par la suite, au vers 17: «Cinq ans sans r'cevoir de nouvelles", puis au vers 35 : «Entre les quatre murs et le lit trop dur» $(4+1)$. Enfin, au vers $60:$ «t c'est déjà le mois de mai » ou le cinquième mois! La dernière strophe est d'ailleurs la seule à avoir cinq vers, comme pour assurer la permanence et la continuité de la verticalité et de l'harmonie. Cette récurrence nous permet de saisir la tentative ultime de coïncidence avec soi d'un être qui a connu, nous le verrons plus tard, le terrorisme du verbe. D'un homme qui cherche à rester debout, envers et contre tout (*Je reviens debout c'est déjà beaucoup", v. 59), d'un être qui, « s'il revient vaincu par la main d'autrui revient vainqueur de lui-même. Ce qui est [...] la plus grande victoire qui se puisse remporter ${ }^{6}$.

Ne pas avoir d'histoire constitue le drame social de la folie. Être soi-même sa propre légende en est la victoire, car le principe de la folie consiste à réaliser une expérience intérieure comme s'il s'agissait d'une expérience extérieure. Il consiste à donner vie, par l'errance. Divaguer? Plutôt errer à l'aventure. Et par l'aventure, tout peut a(d)venir. Le Don Quichotte de Cervantes est un lecteur abusé par les mots, «lui-même à la ressemblance des signes. Long graphisme, maigre comme une lettre, il vient d'échapper tout droit au baillement des livres [...] c'est l'écriture errant dans le monde " ${ }^{7}$. Celui de Rivard se présente en une seule phrase,

5 Jean Chevalier et Alain Gheerbrant, Dictionnaire des symboles, Paris, Laffont-Jupiter, 1982, article * Cinq ".

6 Michel de Cervantes, L'ingénieux hidalgo Don Quichotte de la Manche, Tome II, Paris, Garnier-Flammarion, 1960, p. 483.

7 Michel Foucault, Les mots et les choses, Paris, Gallimard, 1966, p. 60. 
88

indiquant bien qu'il est le héros du semblable, le même Don Quichotte, toujours aux prises avec les mots, avec l'écriture plus précisément: «Je t'écris ces quelques lignes» (v. 1).

$\mathrm{Au}$ chapitre de la folie on trouve évidemment la passion. Ici, celle de l'imaginaire et des mots, des mots écrits, puisque "dire « est un leurre: «J' te raconte pas [...]/ tout c' qu'on m'a fait dire / Ni tout c' qu'on m'a dit pour me fair' croire / Qu'on voulait me guérir * (v. 10-12. C'est moi qui souligne). Le fauxfuyant de la parole est fort bien exprimé par ces deux vers. Alors, si la parole est leurre du cri ( $\star$ Comm' des sirèn's dans la nuit , v. 42), la chanson deviendra l'heure du silence, de l'écrit, de cette écriture qui prend forme dans l'alinéa de la raison: "Écrire ne peut aller sans se taire; écrire, c'est d'une certaine façon, se faire "silencieux comme un mort". 8

On aura saisi l'inefficacité de la parole pour se dire là où nous sommes véritablement. L'écriture, elle, permet une intimité plus grande, un relire qui s'apparente au délire, au lire autrement. De même, la chanson devient le «cheval * de l'écriture, sa locomotion, sa permission: «Tu peux dir' à tout l' monde / Que Don Quichotte est revenu / Avec son ch'fal de porcelaine » (v. 26-27. C'est moi qui souligne). Par cette permission, la parole prend place autrement et ailleurs que dans une errance stérile. C'est parce que le langage appartient à l'instantané, à l'immédiat, qu'il est fugace et sans profondeur. Il berne et illusionne (v. 10-12). Le retour de Don Quichotte évite le piège du présent en se faisant réminiscence du passé. Par le biais de la chanson, Don Quichotte relie fugacité et éternité. Il réinscrit * dans * ce qui n'était que * surface *. Aussi, il se présente a nous brisé, disloqué, * Avec son ch'fal de porcelaine / Et une armure qui ne tient plus * (v. 27-28), s'il nous revient nu (*Don Quichotte est (reve)nu*, v. 26), il n'en est pas moins aussi fort et courageux qu'avant: romances, moulins et géants * Ne lui font pas plus peur qu'avant* (v. 32). De plus, il a (encore) en mains tout le possible du rêve: du cheval à l'armure, en passant par les moulins à vent bien sûr, car dans : «Ne lui font pas plus peur qu'avant *, on entend très bien « à vent», ce qui nous permet de saisir la profondeur d'un imaginaire qui a traversé la surface, * Avant *, c'était hier, mais c'est encore et surtout * à vent *. Le rêve persiste, intègre et intégré. 
"Je ne crois pas qu'on écrive pour "survivre", ni pour se prolonger, ni pour laisser une trace, une "cicatrice" dans la littérature. On écrit, au plus modeste, pour continuer à vivre. ${ }^{9}$ On écrit pour laisser vivre en nous l'être de l'excès, si bien représenté par Don Quichotte qui traverse la littérature et le temps. La chance de l'écriture consiste à toucher la blessure ("On écrit aux larmes sur les draps blancs", v. 37), pour mieux frôler le bord, le vertige. On écrit pour mettre à nu le "dedans", pour exprimer sa douleur et sa passion. La folie consiste alors en un trop-plein de sens, jamais en un $*$ insensé». Hegel dit que l'on "sacrifie son existence au mot", mais au même moment, on la consacre par le mot: « Tu peux dir' à tout l' monde / Que Don Quichotte est revenu * (v. 28-29).

Le retour de Don Quichotte inscrit le passé comme point de départ et d'arrivée. Ici, la réminiscence du passé ne le reconstitue pas, elle le recrée, elle est œuvre (Don Quichotte) et elle œuvre (le retour). Par le passé, tout redevient possible. "Le passé, ce n'est pas ce qui s'est passé, mais ce qui sans cesse se passe et nous passe, ce qui sans cesse se répète en tant que présent disparu. "10 En définitive, le temps du Retour se saisit comme une obsession, dès le titre, à la recherche de ce présent porté disparu, après cinq années d'isolement ( "Cinq ans sans r'cevoir de nouvelles ", v. 17). L'écriture et le «Je» auront pour tâche de dessiner les traces d'une origine qui, peut-être, n'a jamais eu de commencement. Si « devant» reste incertain, "derrière ", tout est possible, parce que "derrière ", il y a autrui (Don Quichotte) et que, comme le dit si bien Edmond Jabès, * autrui est une fiction *

Quand le souvenir s'installe pour laisser place au présent, l'exil s'installe avec lui. C'est par le passé que, toujours, le principe d'identification reprend sa force. Aussi, nous remarquons que le scripteur qui écrit en « $\mathrm{Je}$ " pour parler du présent ( Je t'écris », v. 1), se présente en «il » au refrain, pour nous parler du passé: «Don Quichotte est revenu / Avec son ch'fal de porcelaine* (v. 26-27). Le «Je* qui était île, isolement, devient l'«il », mais l'*il» comme «Je», puisqu'ici l'autre est le même. On peut supposer que l'ex-il est terminé à partir du moment où la fiction (Don Quichotte) se réinstalle (est revenu).

9 François Nourissier, La littérature et la mort ", Magazine littéraire, n 197 juillet-aout 1983, p. 14

10 Shoshana Felman, La folie et la chose littéraire, Paris, Seuil, 1978, p. 110. 
Le retour du passé permet au «Je * altéré de redécouvrir son altérité dans un ailleurs ( $* \mathrm{il} *)$, égal au même ( $* \mathrm{Je} *)$, dans une éternité qui cesse d'être *ternité». Le *Je* qui a poursuivi le rêve quichottesque a travaillé dans l'inter-dit d'une constante infidélité au réel. Par ce travail et ce périple, "il " est revenu au "même*, rendant cette infidélité si... fidèle. En définitive, la perte (exil) se transforme en porte ouverte sur un monde invisible, celui de l'imaginaire. Nier le temps pour n'en garder que les pouvoirs de réminiscence deviendra le but de ce «Je" qui a besoin, pour vivre ou survivre, de nous faire savoir qu'il existe encore. D'où l'importance de ces vers: «Tu peux dir' à tout l' monde / Que Don Quichotte est revenu » (v. 25-26). L'éventualité de l'échec d'une rencontre ( "On pourrait peut-êtr' se voir un peu / Peut-êtr' que t'as pas l' temps», v. 23-24) finira d'installer le «Je * à la troisième personne, là où tout dialogue et toute altérité sont permis.

Dans cette optique, le dialogue avec * Tu $*$ ne représente plus une nécessité, sauf à permettre une représentation de la fiction: «Tu peux dir' à tout l' monde / Que Don Quichotte est revenu » (v. 25-26). «Je * donne la parole à « Tu *, parole qui se veut efficace puisque gratuite. Parole qui réinvente l'histoire passée. En ce sens, parler ou raconter, ce sera dresser la parole au comble de son expérience, faisant une fois de plus du sujet du discours une légende, lui réassurant une éternité, par le biais même du langage. Nous assistons donc à une réussite du langage parlé, en ce sens que ce n'est plus lui qui opère, mais bel et bien le processus de l'imaginaire qui prend place et travaille. L'acharnement à ne pas se laisser saisir par le réel marque bien l'intention de ce Don Quichotte moderne.

Don Quichotte semble encore en quête d'un espace blanc, d'un temps non encombré, où s'inscrire comme personne, où se créer comme identité: "On écrit aux larmes sur les draps blancs * (v. 37). C'est toujours par l'écriture que le *Je* peut s'affirmer et exister. Aussi, il écrit pour lui, pour les fous, pour * dire * et permettre de dire: * Tu peux dir' à tout l' monde * (v. 25). C'est ainsi que naissent les œuvres: de ce rêve des profondeurs qui toujours se poursuit, sans égard à la mort potentielle du sujet (ici Don Quichotte). À la fin du Retour (y a-t--il une fin au retour?), on se trouve à peine plus loin qu'au début, entre l'être et le non-être, entre la parole et le silence: «Et si t'as l' goût d' me voir / J' te racont'rai de bell's histoires / De chevalier (v. 63-65). En attendant? La patience 
$\mathrm{du}$ silence, cette coïncidence qui permet si justement au « Je" d'être par le biais de l'«il". Dans cette bordure du réel, "Je" trouve et institue le jeu où s'abstraire et se confondre, pour ne se représenter que comme aventure et fiction. L'ímaginaire est issu du manque, mais il est, au même moment, tissu du sens. Par exemple, ces draps blancs (v. 37) sur lesquels jouer l'œuvre et lui donner "sa" raison. La fiction appelle le mot à son secours pour camoufler le vide, "penser » la blessure dans l'en-dedans du silence. La fiction devient parole et trouve un alibi, un ailleurs à la folie. "La folie est une voie de la connaissance, un autre mode d'exploration empirique, tant du monde intérieur que du monde extérieur. " 11

Cette exploration se double d'un autre volet: la musique... car la chanson est paroles et musique. Or, la musique viendra jouer ici le même rôle que l'écriture: éternisation de l'instant, dépassement du temporel, arrachement aux conditions matérielles du monde et de la vie. L'écriture et la musique poursuivent le rêve de l'homme, sur la ligne du réel. Comme l'histoire du "Je», la musique s'inscrit dans un temps sans frontières. Elle lui donne un langage spécifique. En effet, alors que le temps réel altère les êtres, le temps rythmique se déploie et les fait renaître, sorte de dé-dramatisation de l'existence, qui nous rappelle que "la musique réussit, dans un laps de temps relativement bref, ce à quoi la vie elle-même ne parvient pas toujours [...] simulant en raccourci cette exaltation de l'accomplissement total 12 . Chant et musique procèdent d'un revenir, d'un retour aux sources, précisément. L'accomplissement total correspond bien à cette intimité et cette coïncidence qui font advenir le "Je * comme égal au «même", qui lui donnent, par emprunt, une identité personnelle.

Le retour de Don Quichotte, nous l'avons déjà noté, est composé en gamme de do majeur et il utilise largement son homonyme en do mineur. La gamme utilisée par Rivard a onze notes, soit: do, $r e ́, m i, f a, f a$ dièse, sol, la bémol, la, si bémol, si. L'emprunt des sonorités mineures par la gamme majeure permet un plus large éventail sonore, comme nous le percevons à l'audition de la chanson. Cependant, il est aussi révélateur de l'emprunt textuel du * Je * au «il * (Don Quichotte). Ce phénomène se répercute à plus d'un niveau

11 Masud Khan, Passion, solitude et folie, Paris, Gallimard, 1983, p. 104.

12 Claude Lévi-Strauss, Mythologiques, t. IV, L'homme nu, Paris, Plon, 1971, p. 587. 
dans Le retour. Ainsi, nous pouvons noter la présence «suspecte " d'un $f a$ dièse dans la gamme de onze notes. Ce fa dièse qui n'est, en définitive, qu'un fa augmenté d'un demi-ton, peur provenir de l'accord du cinquième degré de la dominante de $d o$. Il est donc, à son tour, emprunté à ce système ${ }^{13}$. Au plan textuel, le fa dièse touche le terme «nu * de « revenu * (v. 26), permettant de saisir la mise à nu comme une évidence sonore qui toucherait les deux aspects : le fa et le «Je».

Si l'homonyme de do majeur sert l'auteur-compositeur au plan sonore, il le sert encore au plan textuel, puisque «Je " se sert d'«il» pour exprimer sa réalité. Nous fonctionnons donc sur une double modalité de l'emprunt, du do majeur au do mineur et du «Je» au *il». Cette récurrence de l'emprunt se constate aussi dans le son qui apparaçit au refrain et qui sert d'intermède entre les strophes 8 et 9 . Il s'agit d'un son d'accordéon français, emprunté à un clavier (synthétiseur). Texte et musique se conjuguent donc au même mode (l'emprunt) et au même temps (le passé), pour mieux laisser revenir, a(d)venir Don Quichotte. Il faut également noter la permanence du piano comme support mélodique de toute cette pièce. Il ouvre seul la chanson et la couvre, toujours seul, jusqu'à la septième strophe, moment où apparaissent violons et batterie. Lors de l'intermède musical (entre le refrain $b$ et la strophe 9), tous les instruments disparaissent, sauf le piano et son homonyme (le clavier) d'où sont tirés les sons d'accordéon. Dépouillement du style dans cet accompagnement qui se veut solidaire de l'œuvre, pour mieux en représenter l'état * solitaire ", celui de Don Quichotte. L'importance du piano peut aussi se manifester dans le sens italien du mot, soit: *doucement ». La chanson tout entière baigne dans cette atmosphère de douceur, de calme triste. Comme le retour de Don Quichotte, elle se déroule *calmement * (tel que précisé par Rivard dans sa présentation). La tristesse et la lenteur-langueur qui baignent Don Quichotte ne sont donc pas les seuls fruits des bémols et du type d'accompagnement, mais encore et surtout de cette alliance entre l'histoire et la musique. Alliance qui permet véritablement au * Je* d'être *il ", au fugace de s'installer dans cette éternité venue (re-venue) d'ailleurs. Car, véritablement, l'éternité est. Ailleurs.

13 Nous remercions madame Manon Asselin, professeure de musique, qui nous a fourni les éléments nécessaires à l'analyse de la partie musicale. 